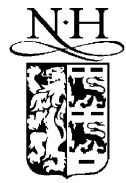

ELSEVIER

\title{
Supertubes and special holonomy
}

\author{
Nicolás E. Grandi ${ }^{1}$, Adrián R. Lugo ${ }^{2}$ \\ Departamento de Física, Facultad de Ciencias Exactas, Universidad Nacional de La Plata, C.C. 67, 1900 La Plata, Argentina
}

Received 12 November 2002; accepted 11 December 2002

Editor: L. Alvarez-Gaumé

\begin{abstract}
We obtain a (1/4)-supersymmetric 6-brane solution of IIA supergravity by T-dualizing the supertube recently found. The resulting $C_{1}$ electric charge is related to the original D0-brane charge. The uplifted solution to eleven dimensions results to be a purely geometrical configuration, which can be interpreted as a bound state of a Taub-NUT space and a pp-wave. Being the non-trivial part of the metric pseudo-Riemannian, the resulting reduced holonomy group is non-compact and locally isomorphic to a semidirect product of an Abelian four-dimensional group and $S U(2)$.

(c) 2002 Elsevier Science B.V. All rights reserved.
\end{abstract}

\section{Introduction}

Tubular configurations of D2-branes preserving some supersymmetries became a subject of interest in recent years [1-6]. The supertube configuration of circular cross section was introduced in [1] from the world-volume point of view, and its corresponding supergravity solution was presented in [2]. In [3] the generalization to arbitrary cross section was found.

The supertube has zero D2 charge, which agrees with its interpretation as the "blown-up" configuration arising when D0 charge is dissolved in a bundle of F1-superstrings. Being (1/4)-supersymmetric, the blown-up configuration is self supported against collapse.

The T-dual configuration along the longitudinal axis of the supertube has been presented in [5]; from the author's analysis of the boundary conditions arising on a superstring that ends on the supertube the picture of a "super-Dhelix" emerged. The ST-dual of the super-D-helix was studied in [6] and identified with the type IIA supercurve, i.e., a string with an arbitrary transverse displacement traveling at the speed of light.

In the present Letter a D6-brane configuration is obtained by T-dualizing the supertube. Somewhat surprisingly, it can be uplifted to a purely geometrical eleven-dimensional solution, that in view of the supersymmetries preserved by the original supertube has a special holonomy group.

E-mail addresses: grandi@fisica.unlp.edu.ar (N.E. Grandi), lugo@fisica.unlp.edu.ar (A.R. Lugo).

1 Fellow CONICET, Argentina.

2 Partially supported by CONICET, Argentina. 


\section{The supertube solution}

The supergravity solution sourced by $N$ coincident supertubes with arbitrary cross section is given by [3]

$$
\begin{aligned}
& d s_{10}^{2}=-V^{-1 / 2} U^{-1}(d t-A)^{2}+V^{1 / 2}\left(U^{-1} d z^{2}+d \vec{y} \cdot d \vec{y}+d \vec{x} \cdot d \vec{x}\right), \\
& B_{2}=-U^{-1}(d t-A) \wedge d z+d t \wedge d z, \\
& C_{1}=V^{-1}(d t-A)-d t, \quad C_{3}=-U^{-1} d t \wedge d z \wedge A, \quad e^{\phi}=U^{-1 / 2} V^{3 / 4},
\end{aligned}
$$

where the coordinates of ten-dimensional spacetime are $(t, z, \vec{y}, \vec{x})$. The supertube we will consider has the topology $\mathcal{C} \times \mathbf{R}$ where the $\mathbf{R}$ direction is identified with the $z$ variable and $\mathcal{C}$ is an arbitrary curve embedded in the $\vec{y}=\left(y_{1}, y_{2}, y_{3}\right)$ hyperplane; $\vec{x} \in \mathbf{R}^{5}$ are coordinates transverse to the supertube.

The harmonic functions $U(\vec{x}, \vec{y}), V(\vec{x}, \vec{y})$ and the 1 -form $A=\vec{A}(\vec{x}, \vec{y}) \cdot d \vec{y}$ are given by:

$$
\left.\begin{array}{l}
U(\vec{x}, \vec{y})-1 \\
V(\vec{x}, \vec{y})-1 \\
\vec{A}(\vec{x}, \vec{y})
\end{array}\right\}=\frac{1}{6 \Omega_{7}} \int_{\mathcal{C}} d \sigma \frac{1}{R(\vec{x}, \vec{y} ; \sigma)^{6}}\left\{\begin{array}{l}
|\pi(\sigma)| \\
|B(\sigma)| \\
N \vec{y}^{\prime}(\sigma)
\end{array},\right.
$$

where we have defined $R(\vec{x}, \vec{y} ; \sigma)=\sqrt{(\vec{y}-\vec{y}(\sigma)) \cdot(\vec{y}-\vec{y}(\sigma))+\vec{x} \cdot \vec{x}}$, and the cross section $\mathcal{C}$ of the supertube is specified by the functions $\vec{y}=\vec{y}(\sigma)$, the F1-string and D0-brane charge densities are denoted $\pi(\sigma)$ and $B(\sigma)$, respectively. Here $\Omega_{q}$ is the volume of a $q$-sphere of unit radius.

The gauge-invariant field-strengths derived form the Neveu-Schwarz $B_{2}$ and Ramond-Ramond $C_{p}$ forms are given by

$$
H_{3}=d B_{2}, \quad G_{2}=d C_{1}, \quad G_{4}=d C_{3}+H_{3} \wedge C_{1} .
$$

For the solution (1) they read

$$
\begin{aligned}
& H_{3}=U^{-2} d U \wedge(d t-A) \wedge d z+U^{-1} d A \wedge d z \\
& G_{2}=-V^{-2} d V \wedge(d t-A)-V^{-1} d A, \\
& G_{4}=-U^{-1} V^{-1}(d t-A) \wedge d z \wedge d A .
\end{aligned}
$$

The Hodge duals of the above RR-curvatures are

$$
\begin{aligned}
& G_{6} \equiv *_{10} G_{4} \equiv d C_{5}+H_{3} \wedge C_{3}=*_{8} d A, \\
& G_{8} \equiv *_{10} G_{2} \equiv-d C_{7}-H_{3} \wedge C_{5}=\left(-U^{-1} G_{6} \wedge(d t-A)-*_{8} d V\right) \wedge d z,
\end{aligned}
$$

where $*_{8}$ stands for the eight-dimensional Hodge dual with respect to the Euclidean $(\vec{y}, \vec{x})$ space and the dual potentials, defined according to the equations of motion, ${ }^{3}$ are given by

$$
\begin{aligned}
& C_{5}=*_{8} T_{3}, \\
& C_{7}={ }_{8} T_{2} \wedge d z+U^{-1} C_{5} \wedge(d t-A) \wedge d z,
\end{aligned}
$$

where we have introduced the two new forms $T_{2}$ and $T_{3}$ that must satisfy the constraints

$$
d *_{8} T_{3}=*_{8} d A, \quad d *_{8} T_{2}=*_{8} d V .
$$

\footnotetext{
3 We follow conventions of [7].
} 


\section{T-duality}

Let $\left(x^{\mu}, y\right)$ be coordinates in which a $y$-translation is a symmetry of the whole background (metric $G_{M N}$, antisymmetric tensor $B_{M N}$, dilaton $\phi$, RR fields $\left.C_{p}\right)$ and $\left(x^{\mu}\right)$ coordinates in nine spacetime dimensions. Then to perform a T-duality in $y$-direction we use the following form of the duality rules; on NS-NS fields

$$
\begin{aligned}
& \widetilde{d s}_{10}^{2}=d s_{9}^{2}+G_{y y}^{-1}(b+d y)^{2}-G_{y y} g^{2}, \\
& \widetilde{B}_{2}=\left.B_{2}\right|_{9}+g \wedge(b+d y), \quad e^{\tilde{\phi}}=G_{y y}^{-1 / 2} e^{\phi},
\end{aligned}
$$

where $g \equiv G_{y y}^{-1} G_{\mu y} d x^{\mu}, b \equiv B_{\mu y} d x^{\mu}$ and $\left.B_{2}\right|_{9}=B_{\mu \nu} d x^{\mu} \wedge d x^{\nu}$, and on RR fields

$$
\widetilde{C}_{n}=\left(\partial_{y} ; C_{n+1}\right)+\left(\partial_{y} ; C_{n-1}\right) \wedge(b+d y) \wedge g+\left(\partial_{y} ; C_{n-1} \wedge d y\right) \wedge b+C_{n-1} \wedge d y,
$$

where $\left(u ; t_{n+1}\right)_{\mu_{1} \ldots \mu_{n}} \equiv u^{\mu} t_{\mu_{1} \ldots \mu_{n} \mu}$.

We will use these rules to T-dualize the solution (1) in the directions $(z, \vec{x})$. After the first T-duality in the $z$ direction, the resulting non-zero fields are

$$
\begin{aligned}
& d s_{10}^{2}=V^{-1 / 2}\left((U-1) d z^{+^{2}}-d z^{+}\left(d z^{-}-2 A\right)\right)+V^{1 / 2}(d \vec{y} \cdot d \vec{y}+d \vec{x} \cdot d \vec{x}), \\
& C_{2}=\frac{1}{2}\left(V^{-1}\left(d z^{-}-2 A\right)-d z^{-}\right) \wedge d z^{+}, \quad C_{6}=C_{5} \wedge d z^{+}+*_{8} T_{2}, \quad e^{\phi}=V^{1 / 2},
\end{aligned}
$$

where $z^{ \pm}=t \pm z, C_{5}$ and $T_{2}$ are given in (6) and (7), respectively. This supergravity solution corresponds to the super-D-helix first studied in [5], the ST-dual of which is the supercurve of [6]. Here we see that the $B_{2}$ form vanishes. This is due to the fact that the F-strings extending in the $z$ direction are transformed into light-like momentum modes in the $z$ direction after the T-dualization.

In order to perform a T-duality in a given direction, the fields have to be independent of the corresponding variable. This is achieved by delocalizing the solution, i.e., forming an array of supertubes in the direction to be T-dualized and taking the limit in which the separating distance goes to zero and the charge densities remain constant. For each one the $\vec{x}$ directions, the net effect of these operations in (2) is to decrease the power of $R(\sigma)$ in one, eliminating the corresponding dependence. The $(q-1) \Omega_{q}$ denominator in the solutions goes to $(q-2) \Omega_{q-1}$ on each step. After delocalizing the supertube in the five $\vec{x}$ directions we obtain

$$
\left.\begin{array}{l}
U(\vec{y})-1 \\
V(\vec{y})-1 \\
\vec{A}(\vec{y})
\end{array}\right\}=\frac{1}{4 \pi} \int_{\mathcal{C}} d \sigma \frac{1}{|\vec{y}-y(\sigma)|}\left\{\begin{array}{l}
|\pi(\sigma)| \\
|B(\sigma)| \\
N \vec{y}^{\prime}(\sigma)
\end{array} .\right.
$$

It will be convenient in what follows to rewrite the $T_{2}$ and $T_{3}$ forms in terms of their 3-dimensional Hodge duals w.r.t. the $\vec{y}$ variables, $T_{1}=*_{3} T_{2}=\vec{T} \cdot d \vec{y}$ and $T=*_{3} T_{3}$, then the constraints (7) are written as

$$
\vec{\nabla} T=\vec{\nabla} \times \vec{A}, \quad \vec{\nabla} \times \vec{T}=\vec{\nabla} V .
$$

Next we will T-dualize (10) in the five $\vec{x}$ directions, which are transverse to the supertube. We obtain

$$
\begin{aligned}
& d s_{10}^{2}=V^{-1 / 2}\left((U-1) d z^{+^{2}}-d z^{+}\left(d z^{-}-2 A\right)+d \vec{x} \cdot d \vec{x}\right)+V^{1 / 2} d \vec{y} \cdot d \vec{y}, \\
& C_{1}=T_{1}-T d z^{+}, \quad C_{7}=\frac{1}{2}\left(V^{-1}\left(d z^{-}-2 A\right)-d z^{-}\right) \wedge d z^{+} \wedge d x_{1} \wedge \cdots \wedge d x_{5}, \\
& e^{\phi}=V^{-3 / 4} .
\end{aligned}
$$

Let us remark here that the NS 2-form $B_{2}$ and the RR 3-form $C_{3}$ vanish, which implies that this solution can be uplifted to a purely geometrical eleven-dimensional configuration, as we will see in the next section. 


\section{Eleven-dimensional metric and reduced holonomy}

\subsection{Uplifting to eleven dimensions}

Let us carry out the uplifting to $D=11$ of the D6-brane like solution of $D=10$ SUGRA IIA just found, according to the standard recipe

$$
d s_{11}^{2}=e^{-\frac{2}{3} \phi} d s_{10}^{2}+e^{\frac{4}{3} \phi}\left(d \psi+C_{1}\right)^{2}, \quad A_{3}=C_{3}+B_{2} \wedge d \psi
$$

where $\psi$ is the eleventh dimension. Being $C_{3}=B_{2}=0$ we get $A_{3}=0$, i.e., the solution is a pure geometric one. It reads

$$
\begin{aligned}
& d s_{11}^{2}=d s_{1,5}^{2}+d \vec{x} \cdot d \vec{x}, \\
& d s_{1,5}^{2}=\left(U-1+V^{-1} T^{2}\right) d z^{+^{2}}-d z^{+}\left(d z^{-}+\mathcal{A}\right)+V d \vec{y} \cdot d \vec{y}+V^{-1}\left(d \psi+T_{1}\right)^{2},
\end{aligned}
$$

where $\mathcal{A}=-2 A+2 V^{-1} T\left(d \psi+T_{1}\right)$ is the so-called Sagnac connection [8].

It will be convenient in what follows to work in a local basis, so let us introduce the following elfbein $\left\{\omega^{A}\right\}$ together with the dual basis $\left\{e_{A}\right\}$ in $T(M),\left(e_{A} ; \omega^{B}\right)=\delta_{A}^{B}$,

$$
\begin{array}{ll}
\omega^{0}=U^{-1 / 2}(d t-A), & e_{0}=U^{1 / 2}\left(\partial_{t}-\left(1-U^{-1}\right) \partial_{z}+U^{-1} T \partial_{\psi}\right), \\
\omega^{i}=V^{1 / 2} d y^{i}, & e_{i}=V^{-1 / 2}\left(\partial_{i}+A_{i}\left(\partial_{t}-\partial_{z}\right)-T_{i} \partial_{\psi}\right), \\
\omega^{4}=V^{-1 / 2}\left(d x^{10}+C_{1}\right), & e_{4}=V^{1 / 2} \partial_{\psi}, \\
\omega^{5}=U^{-1 / 2}(U d z+(U-1) d t+A), & e_{5}=U^{-1 / 2}\left(\partial_{z}+T \partial_{\psi}\right), \\
\omega^{a}=d x^{a-5}, & e_{a}=\partial_{x^{a-5}},
\end{array}
$$

where $i=1,2,3$ and $a=6, \ldots, 10$ and $C_{1}$ is given in (13). The metric (15) is simply

$$
d s_{11}^{2}=\eta_{A B} \omega^{A} \omega^{B}, \quad \eta=\operatorname{diag}(-1,1, \ldots, 1) .
$$

The (pseudo-) Riemannian connection is a one-form valued (in general) in the $\operatorname{spin}(1,10)$ algebra

$$
\Omega \equiv \frac{1}{2} \omega^{A B} X_{A B}, \quad\left[X_{A B} ; X_{C D}\right]=\eta_{A D} X_{B C}+\eta_{B C} X_{A D}-(A \leftrightarrow B),
$$

and its components are fixed by the conditions of metricity and no-torsion

$$
\omega^{A B}=-\omega^{B A}, \quad d \omega^{A}+\omega^{A B} \wedge \omega_{B}=0 .
$$

For the metric (17) we get the following non-zero components

$$
\begin{aligned}
& \omega_{0 i}=\frac{1}{2} V^{-1} U^{-1 / 2}\left(V^{1 / 2} U^{-1 / 2} \partial_{i} U\left(\omega^{0}+\omega^{5}\right)+\epsilon_{i j k} B_{k} \omega^{j}-B_{i} \omega^{4}\right)=-\omega_{i 5}, \\
& \omega_{04}=-\frac{1}{2} V^{-1} U^{-1 / 2} B_{i} \omega^{i}=-\omega_{45}, \\
& \omega_{05}=\frac{1}{2} V^{-1 / 2} U^{-1} \partial_{i} U \omega^{i}=d \ln U^{1 / 2}, \\
& \omega_{i 4}=\frac{1}{2} V^{-3 / 2}\left(V^{1 / 2} U^{-1 / 2} B_{i}\left(\omega^{0}+\omega^{5}\right)+\epsilon_{i j k} \partial_{j} V \omega^{k}+\partial_{i} V \omega^{4}\right)=-\frac{1}{2} \epsilon_{i j k} \omega_{j k},
\end{aligned}
$$

where $B_{i}=\epsilon_{i j k} \partial_{j} A_{k}$ and indices are raised and lowered with $\left(\eta_{A B}\right)$.

The curvature tensor is a two-form valued (in general) in $\operatorname{spin}(1,10)$

$$
\begin{aligned}
& R \equiv d \Omega+\Omega \wedge \Omega=\frac{1}{2} R^{A B} X_{A B}, \\
& R_{A B} \equiv d \omega_{A B}+\omega_{A C} \wedge \omega_{B}^{C}=\frac{1}{2} R_{A B C D} \omega^{C} \wedge \omega^{D} .
\end{aligned}
$$


It is straightforwardly computed from (16), (20); we get ${ }^{4}$

$$
\begin{aligned}
R_{0 i}= & \frac{1}{4} U^{-1} V^{-2}\left(\left(4 B_{i} B_{j}+\delta_{i j}\left(\vec{\nabla} U \cdot \vec{\nabla} V-\vec{B}^{2}\right)+2 V \partial_{i} \partial_{j} U\right.\right. \\
& \left.\left.\quad-\partial_{i} U \partial_{j} V-\partial_{j} U \partial_{i} V\right) \omega^{j}-\epsilon_{i j k} \partial_{j} U \partial_{k} V \omega^{4}\right) \wedge\left(\omega^{0}+\omega^{5}\right) \\
& +\frac{1}{2} U^{-1 / 2} V^{-5 / 2}\left(\vec{B} \cdot \vec{\nabla} V \delta_{i l}-2 B_{i} \partial_{l} V-B_{l} \partial_{i} V+V \partial_{i} B_{l}\right) \omega^{4} \wedge \omega^{l} \\
& +\frac{1}{2} \epsilon_{l j k}\left(R_{0 i 4 l}+\frac{1}{2} U^{-1 / 2} V^{-3 / 2} \epsilon_{l i m} \nabla^{2} A_{m}\right) \omega^{j} \wedge \omega^{k}, \\
R_{04}= & \frac{1}{4} U^{-1} V^{-2}\left(-\left(\vec{B}^{2}+\vec{\nabla} U \cdot \vec{\nabla} V\right) \omega^{4}-\epsilon_{i j k} \partial_{j} U \partial_{k} V \omega^{i}\right) \wedge\left(\omega^{0}+\omega^{5}\right) \\
& -\frac{1}{2} \epsilon_{i j k}\left(R_{k 404}-\frac{1}{2} U^{-1 / 2} V^{-3 / 2} \nabla^{2} A_{k}\right) \omega^{i} \wedge \omega^{j}, \\
R_{05}= & 0, \\
R_{i 4}= & \left(\left(-R_{0 j i 4}+\frac{1}{2} U^{-1 / 2} V^{-3 / 2} \epsilon_{i j k} \nabla^{2} A_{k}\right) \omega^{j}-\frac{1}{2} U^{-1 / 2} V^{-5 / 2} \epsilon_{i j k} B_{j} \partial_{k} V \omega^{4}\right) \wedge\left(\omega^{0}+\omega^{5}\right) \\
& +\frac{1}{2} V^{-3}\left(\vec{\nabla} V \cdot \vec{\nabla} V \delta_{i j}-3 \partial_{i} V \partial_{j} V+V \partial_{j} \partial_{i} V\right) \omega^{j} \wedge \omega^{4}-\frac{1}{2} \epsilon_{l j k}\left(R_{l 4 i 4}-\frac{1}{2} V^{-2} \nabla^{2} V \delta_{l i}\right) \omega^{j} \wedge \omega^{k},
\end{aligned}
$$

while the other components are obtained from the relations

$$
R_{i 5}=-R_{0 i}, \quad R_{45}=-R_{04}, \quad R_{i j}=-\epsilon_{i j k} R_{k 4} .
$$

The non-zero components of the Ricci tensor $\mathcal{R}_{A B} \equiv R^{C}{ }_{A C B}=\mathcal{R}_{B A}$ and Ricci scalar result

$$
\begin{aligned}
& \mathcal{R}_{00}=-\frac{1}{2} V^{-1} U^{-1} \nabla^{2} U=\mathcal{R}_{55}, \quad \mathcal{R}_{i j}=-\mathcal{R}_{44} \delta_{i j}, \\
& \mathcal{R}_{44}=\frac{1}{2} V^{-2} \nabla^{2} V, \quad \mathcal{R} \equiv \mathcal{R}^{A}{ }_{A}=-V^{-2} \nabla^{2} V .
\end{aligned}
$$

\footnotetext{
${ }^{4}$ It is worth to note a subtlety that arises when "magnetic" sources coupled to gravity are present. While the properties of the curvature tensor $R_{A B C D}=-R_{A B D C}=-R_{B A C D}$ follow from the two-form condition and metricity, respectively, more or less by definition, the torsionless condition in (19) leads to the anomaly relation

$$
R_{A B C D}-R_{C D A B}=-\frac{1}{2}\left(d_{A B C D}-d_{C D A B}+d_{D A B C}-d_{B C D A}\right)
$$

where we have defined

$$
d^{2} \omega^{A} \equiv \frac{1}{3 !} d_{B C D^{\omega}}^{A} \wedge \omega^{C} \wedge \omega^{C}, \quad d_{B C D}^{A} \equiv e_{B}^{M} e_{C}^{N} e_{D}^{P}\left(\left[\partial_{M} ; \partial_{N}\right] \omega_{P}^{A}+\left[\partial_{P} ; \partial_{M}\right] \omega_{N}^{A}+\left[\partial_{N} ; \partial_{P}\right] \omega_{M}^{A}\right) .
$$

In "normal" conditions (when the vielbein is smooth enough such that derivatives commute on them) the coefficients $\left\{d_{A B C D}\right\}$ are identically null and the textbook property $R_{A B C D}=R_{C D A B}$ holds; however, when "Dirac string" like singularities are present its generalization (22) must be considered. In the case at hand (16), (17) it can be shown by using (12) that the non-trivial anomaly terms are

$$
\begin{aligned}
& R_{i 4 j k}-R_{j k i 4}=\frac{1}{2} d_{4 i j k}=\frac{1}{2} \epsilon_{i j k} V^{-2} \nabla^{2} V, \\
& R_{04 i j}-R_{i j 04}=\frac{1}{2} d_{40 i j}=\frac{1}{2} \epsilon_{i j k} U^{-1 / 2} V^{-3 / 2} \nabla^{2} A_{k}=R_{54 i j}-R_{i j 54}=\frac{1}{2} d_{45 i j}=R_{4 i 0 j}-R_{0 j 4 i}=R_{4 i 5 j}-R_{5 j 4 i} .
\end{aligned}
$$
}

Explicit computation shows them up in (25). 
This shows that the metric is a solution of $D=11$ SUGRA coupled to an object living on the curve $\mathcal{C}$, being Ricci flat outside it. ${ }^{5}$

\subsection{Holonomy group and Killing spinors}

It is clear that, being reducible, the holonomy group of (15) will be

$$
\operatorname{Hol}\left(d s_{11}^{2}\right)=\operatorname{Hol}\left(d s_{1,5}^{2}\right) \times \operatorname{Hol}(d \vec{x} \cdot d \vec{x})=\operatorname{Hol}\left(d s_{1,5}^{2}\right) \subset \operatorname{Spin}(1,5),
$$

but being the solution (1/4)-supersymmetric (see below for more) the holonomy will be further reduced. To see this let us introduce the light-cone generators $X^{ \pm a} \equiv X^{0 a} \pm X^{5 a}, a=1,2,3,4, X^{+-}=-2 X^{05}$ and in terms of them the following (redundant) ones

$$
\begin{aligned}
& T_{i}^{( \pm)}=\frac{1}{4} \epsilon_{i j k} X_{j k} \pm \frac{1}{2} X_{i 4}, \quad i=1,2,3 \\
& t_{ \pm}^{1}=X^{+3} \mp i X^{+4}, \quad \bar{t}_{ \pm}^{1}=X^{+3} \pm i X^{+4}, \quad t_{ \pm}^{2}=X^{+1}+i X^{+2}, \quad \bar{t}_{ \pm}^{2}=X^{+1}-i X^{+2},
\end{aligned}
$$

leaving aside $\left\{X^{-a}, a=1,2,3,4,+\right\}$ which will not play any role in the discussion. It is easy to check the following commutation relations

$$
\begin{aligned}
& {\left[T_{i}^{( \pm)} ; T_{j}^{( \pm)}\right]=-\epsilon_{i j k} T_{k}^{( \pm)}, \quad\left[T_{i}^{(+)} ; T_{j}^{(-)}\right]=0,} \\
& {\left[T_{i}^{( \pm)} ; t_{ \pm}^{\alpha}\right]=-\frac{i}{2} \sigma_{i}{ }^{\alpha}{ }_{\beta} t_{ \pm}^{\beta}, \quad\left[T_{i}^{( \pm)} ; \bar{t}_{ \pm}^{\alpha}\right]=\frac{i}{2} \sigma_{i}^{* \alpha}{ }_{\beta} \bar{t}_{ \pm}^{\beta},} \\
& {\left[t_{ \pm}^{\alpha} ; t_{ \pm}^{\beta}\right]=\left[t_{ \pm}^{\alpha} ; \bar{t}_{ \pm}^{\beta}\right]=\left[t_{+}^{\alpha} ; t_{-}^{\beta}\right]=\left[t_{+}^{\alpha} ; \bar{t}_{-}^{\beta}\right]=0 .}
\end{aligned}
$$

The first line is just the $\operatorname{spin}(4) \sim s u(2)_{+} \times s u(2)_{-}$subalgebra of $\operatorname{spin}(1,5)$, while the second line indicates that $\left(t_{ \pm}^{\alpha}\right)$ and $\left(\bar{t}_{ \pm}^{\alpha}\right)$ transform in the spin $1 / 2$ representation of $s u(2)_{ \pm}$and its conjugate (equivalent anyway), respectively. Finally the third line implies that $\left\{t_{ \pm}^{\alpha}, \bar{t}_{ \pm}^{\alpha}\right\}$ expand an Abelian subalgebra.

Now from the curvature tensor we can read the Lie algebra $\operatorname{hol}\left(d s_{11}^{2}\right)$ of $\operatorname{Hol}\left(d s_{11}^{2}\right)$; from (25), (26) it takes the form

$$
R=R_{0 i} T_{i}^{(-)}+\frac{1}{2}\left(\left(R_{03}-i R_{04}\right) t_{-}^{1}+\left(R_{01}-i R_{02}\right) t_{-}^{2}+\text { h.c. }\right),
$$

which shows that $\operatorname{hol}\left(d s_{11}^{2}\right)$ is the seven-dimensional subalgebra whose elements can be written as

$$
X=\epsilon_{i} T_{-}^{i}+\epsilon_{\alpha} t_{-}^{\alpha}+\bar{\epsilon}_{\alpha} \bar{t}_{-}^{\alpha}, \quad \epsilon_{i} \in \mathbf{R}, \bar{\epsilon}_{\alpha}=\epsilon_{\alpha}^{*} .
$$

It is given by

$$
\begin{aligned}
& {\left[T_{i}^{(-)} ; T_{j}^{(-)}\right]=-\epsilon_{i j k} T_{k}^{(-)}, \quad\left[T_{i}^{(-)} ; t_{-}^{\alpha}\right]=-\frac{i}{2} \sigma_{i}{ }^{\alpha}{ }_{\beta} t_{-}^{\beta}, \quad\left[T_{i}^{(-)} ; \bar{t}_{-}^{\alpha}\right]=\frac{i}{2} \sigma_{i}^{* \alpha}{ }_{\beta} \bar{t}_{-}^{\beta},} \\
& {\left[t_{-}^{\alpha} ; t_{-}^{\beta}\right]=\left[t_{-}^{\alpha} ; \bar{t}_{-}^{\beta}\right]=\left[\bar{t}_{-}^{\alpha} ; \bar{t}_{-}^{\beta}\right]=0 .}
\end{aligned}
$$

On the other hand, in view of the pure geometric character of the solution we have that the variation of the gravitino field $\chi_{A}^{\Lambda}$ under a SUGRA transformation is just

$$
\delta_{\epsilon} \chi_{A}^{\Lambda}=D_{A} \epsilon^{\Lambda}=e_{A}\left(\epsilon^{\Lambda}\right)+S(\Omega)_{\Lambda^{\prime}}^{\Lambda} \epsilon^{\Lambda^{\prime}},
$$

\footnotetext{
${ }^{5}$ Let us remind the reader that from (11) it follows that

$$
-\nabla^{2}\left\{\begin{array}{l}
U(\vec{y}) \\
V(\vec{y})=\int_{\mathcal{C}} d \sigma \delta^{3}(\vec{y}-\vec{y}(\sigma))\left\{\begin{array}{l}
|\pi(\sigma)| \\
\vec{A}(\vec{y})
\end{array}|B(\sigma)| .\right. \\
\vec{y}^{\prime}(\sigma)
\end{array}\right.
$$
}


where the parameter $\epsilon^{\Lambda}$ is a thirty two component Majorana spinor and $S(\Omega)$ is the connection (18) valued in the spinorial representation. The solution will preserve the SUSY's that annihilate the gravitino variation, so the Killing spinors will be the covariantly constant ones. Specifically in a Weyl basis where for definiteness we take

$$
\Gamma_{m}=\left(\begin{array}{cc}
0_{16} & \gamma_{m} \\
\gamma_{m} & 0_{16}
\end{array}\right), \quad \Gamma_{9}=i\left(\begin{array}{cc}
0_{16} & 1_{16} \\
-1_{16} & 0_{16}
\end{array}\right), \quad \Gamma_{10}=\left(\begin{array}{cc}
1_{16} & 0_{16} \\
0_{16} & -1_{16}
\end{array}\right),
$$

where $\left\{\gamma_{m}, m=0,1, \ldots, 8\right\}$ are gamma-matrices of $\operatorname{spin}(1,8)$, the condition $\delta_{\epsilon} \chi_{A}^{\Lambda}=D_{A} \epsilon^{\Lambda}=0$ results equivalent to

$$
\epsilon=U^{-1 / 4} \epsilon_{0}, \quad \text { with }\left\{\begin{array}{l}
\Gamma_{05} \epsilon_{0}=\epsilon_{0} \\
\Gamma_{1234} \epsilon_{0}=-\epsilon_{0}
\end{array},\right.
$$

with $\epsilon_{0}$ a constant spinor. Eq. (36) signals the preservation of $8=\frac{1}{4} \cdot 32$ of the supersymmetries as it happened for the supertube. It is worth to note that the Killing spinors can be made constant through a Lorentz boost that eliminate the part of the connection that does not lie in the holonomy algebra, given $\omega_{05} S\left(X^{05}\right)=d \ln U^{1 / 2}\left(\Gamma^{05} / 2\right)$.

\section{Discussion}

First let us note that the supersymmetry of the supergravity supertube solution does not depend on any relation between the D0 and F1 charge densities, what enables us to assign arbitrary values to them.

While the original supertube had zero D2 charge, the final ten-dimensional configuration (13) is a genuine D6-brane, its charge being traced to the initial D0-brane density,

$$
q_{\mathrm{D} 6} \equiv \int_{S^{2}} *_{10} F_{8}=\int_{S^{2}} F_{2}=\int_{S^{2}} *_{3} d V=\int_{B_{3}, \partial B_{3}=S^{2}} d *_{3} d V=-\int_{\mathcal{C}} d \sigma|B(\sigma)| .
$$

Of course, for $U=1, \vec{A}=\overrightarrow{0}$ (which means $|\pi(\sigma)|=0$ on a flat D2-brane) we recover the standard flat $1 / 2$ supersymmetric D6-brane solution.

When uplifted to eleven dimensions, the resulting configuration (15) can be interpreted as a bound state of a Taub-NUT like space and pp-wave. The presence of the first can be verified by direct inspection of the metric, while that of the second follows from the existence of the covariantly constant null vector $\partial_{-}$. This assertion is consistent with the analysis of the Killing spinors since the constraints (36) are those corresponding to a Taub-NUT and a pp-wave supersymmetry preserving conditions [9].

For $U=1, \vec{A}=\overrightarrow{0}$ we have the product of Minkowski space in $1+6$ dimensions and a Taub-NUT space, i.e., M-theory compactified on this last one which is known to preserve $1 / 2$ of the supersymmetries and to have $S U(2) \subset G_{2}$ holonomy. Since in our general (1/4)-supersymmetric case the irreducible part of the metric is sixdimensional one could expect to find a $\operatorname{Hol}\left(d s_{1,5}^{2}\right) \subset S U(3)$ reduced holonomy group, but the pseudo-Riemannian character of $d s_{1,5}^{2}$ invalidates Berger's classification theorem [10] and the resulting manifold is not a Calabi-Yau one. ${ }^{6}$ In fact, from (33) the holonomy group is non-semisimple and locally isomorphic to the semidirect product of $S U(2)$ and an the real section defined in (32) of the Abelian four-dimensional algebra generated by $\left\{t_{-}^{\alpha}, \bar{t}_{-}^{\alpha}\right\}$ transforming in the $\frac{1}{2} \oplus \overline{\frac{1}{2}}$.

\section{Acknowledgements}

We would like to thank Carlos Núñez for an enlightening discussion.

\footnotetext{
${ }^{6}$ At the time of writing we have no knowledge about a classification of holonomy groups of pseudo-Riemannian manifolds.
} 


\section{References}

[1] D. Mateos, P.K. Townsend, Phys. Rev. Lett. 87 (2001) 011602, hep-th/0103030.

[2] R. Emparan, D. Mateos, P.K. Townsend, JHEP 0107 (2001) 011, hep-th/0106012.

[3] D. Mateos, S. Ng, P.K. Townsend, JHEP 0203 (2002) 016, hep-th/0112054.

[4] D. Bak, A. Karch, Nucl. Phys. B 626 (2002) 165, hep-th/0110039.

[5] J.H. Cho, P. Oh, Phys. Rev. D 64 (2001) 106010, hep-th/0105095.

[6] D. Mateos, S. Ng, P.K. Townsend, Phys. Lett. B 538 (2002) 366, hep-th/0204062.

[7] C.V. Johnson, hep-th/0007170.

[8] G.W. Gibbons, C.A. Herdeiro, Class. Quantum Grav. 16 (1999) 3619, hep-th/9906098.

[9] J.P. Gauntlett, hep-th/9705011.

[10] D. Joyce, math.DG/0108088. 